\title{
THE MERGER-DRIVEN EVOLUTION OF MASSIVE GALAXIES
}

\author{
Aday R. Robaina ${ }^{1}$, Eric F. Bell ${ }^{2}$, Arjen van der Wel ${ }^{1}$, Rachel S. Somerville ${ }^{3}$, Rosalind E. Skelton ${ }^{1}$, \\ Daniel H. McIntosh ${ }^{4}$, Klaus Meisenheimer ${ }^{1}$, and Christian Wolf ${ }^{5}$

\begin{abstract}
We explore the rate and impact of galaxy mergers on the massive galaxy population using the amplitude of the two-point correlation function on small scales for $M_{*}>5 \times 10^{10} M_{\odot}$ galaxies from the COSMOS and COMBO-17 surveys. Using a pair fraction derived from the Sloan Digital Sky Survey as a low-redshift benchmark, the large survey area at intermediate redshifts allows us to determine the evolution of the closepair fraction with unprecedented accuracy for a mass-selected sample: we find that the fraction of galaxies more massive than $5 \times 10^{10} M_{\odot}$ in pairs separated by less than $30 \mathrm{kpc}$ in three-dimensional space evolves as $F(z)=(0.0130 \pm 0.0019) \times(1+z)^{1.21 \pm 0.25}$ between $z=0$ and $z=1.2$. Assuming a merger timescale of $0.5 \mathrm{Gyr}$, the inferred merger rate is such that galaxies with mass in excess of $10^{11} M_{\odot}$ have undergone, on average, $0.5(0.7)$ mergers involving progenitor galaxies both more massive than $5 \times 10^{10} M_{\odot}$ since $z=0.6(1.2)$. We also study the number density evolution of massive red sequence galaxies using published luminosity functions and constraints on the $\mathrm{M} / \mathrm{L}_{B}$ evolution from the fundamental plane. Moreover, we demonstrate that the measured merger rate of massive galaxies is sufficient to explain this observed number density evolution in massive red sequence galaxies since $z=1$.
\end{abstract}

Key words: galaxies: elliptical and lenticular, $\mathrm{cD}$ - galaxies: evolution - galaxies: formation - galaxies: general galaxies: interactions - galaxies: statistics

Online-only material: color figure

\section{INTRODUCTION}

The distribution of galaxies in a color-magnitude diagram shows a bimodality: galaxies with red optical colors occupy a relatively tight sequence in color (the red sequence), while blue galaxies show a wider dispersion and populate the so-called "blue cloud" (Blanton et al. 2003). This bimodality has existed for most of the history of the universe (e.g., Bell et al. 2004; Faber et al. 2007; Taylor et al. 2009). However, the stellar mass density of red sequence galaxies has increased by a factor of $\sim 2$ since $z=1$, while it has remained approximately constant for blue galaxies (Bell et al. 2004; Faber et al. 2007; Brown et al. 2007). Yet, the majority of stars are formed in blue galaxies (Bell et al. 2007; Walcher et al. 2008), which implies that galaxies migrate from the blue cloud to the red sequence (see, e.g., the discussion by Faber et al. 2007).

While significant evolution of the integrated mass function of red galaxies is generally agreed upon, the evolution in the number density of high-mass galaxies has been shown to be consistent with zero (e.g., Cimatti et al. 2006; Cool et al. 2008). However, given the large uncertainties, the constraints are not tight. There is ample room for considerable evolution, and such evolution is expected in a $\Lambda$ CDM hierarchical cosmology, which predicts significant growth of galaxies through merging, especially at the high-mass end (De Lucia \& Blaizot 2007).

The most massive, non-star-forming galaxies are essentially all spheroidal (i.e., have ellipticities $<0.4$; van der Wel et al. 2009), indicating that they formed through merging (Toomre \& Toomre 1972; Schweizer \& Seitzer 1992; Kormendy et al. 2009). Shape distributions do not specify when merging happened. Some valuable constraints have been placed by the study of the clustering properties of red galaxies in this mass range by White et al. (2007), who found that $1 / 3$ of massive red satellites disappear between $z=0.9$ and $z=0.5$ by merging with the central galaxies in their halos.

The goal of this paper is to estimate the impact of galaxy mergers on the evolution of massive galaxies from $z \sim 1$ to the present day, and more specifically, on the massive end of the red sequence. To that end we measure the merger fraction since $z=1.2$ in a large sample of mass-selected galaxies. We will test whether these measurements are consistent with predictions from hierarchical formation models, and, providing an independent estimate of the number density of the most massive red galaxies, with the observed evolution of the bright tail of the luminosity function (LF) of red galaxies.

It is observationally challenging to identify galaxy mergers, especially at large cosmological distances. Mergers are found either in an early phase of the interaction, when the two galaxies have not yet coalesced and are found in a close pair (Patton et al. 2000; Le Fèvre et al. 2000; Lin et al. 2004, 2008; Bell et al. 2006a; Kartaltepe et al. 2007; Robaina et al. 2009), or in a later phase, when they display signatures of gravitational interaction and are just prior to or after coalescence (Abraham et al. 1996; Conselice et al. 2003; Lotz et al. 2004; Bell et al. 2005; McIntosh et al. 2008; Jogee et al. 2009; Heiderman et al. 2009; Robaina et al. 2009). A wide range of results have been found for the merger fraction evolution, parameterized as $(1+z)^{m}$, with $m$ ranging from 0 to 4 (e.g., Patton et al. 2000; Le Fèvre et al. 2000; Lin et al. 2004; Kartaltepe et al. 2007).

Here, we use robust two-point correlation function (2pcf) techniques on a sample of galaxies with $0.2<z<1.2$ with stellar masses in excess of $5 \times 10^{10} M_{\odot}$ selected from the 
COSMOS and COMBO-17 surveys to quantify the merger rate of massive galaxies and its evolution. We augment the statistical significance of our analysis by using an estimate of the pair fraction found in Sloan Digital Sky Survey (SDSS) at $z \sim 0.1$. The total volume probed by this study at intermediate redshifts is at least four times larger than any previous mass-selected study on the evolution of the merger fraction and reduces dramatically the systematic uncertainties related to cosmic variance by the use of four independent fields. Then we compare the inferred galaxy merger rate with the observed number density evolution of massive $\left(M_{*}>10^{11} M_{\odot}\right)$, red galaxies from $z \sim 1$ to the present day, which we obtain by converting Brown et al. (2007) LFs to stellar-mass functions. We assume $H_{0}=70 \mathrm{~km} \mathrm{~s}^{-1}$, $\Omega_{m}=0.3$, and $\Omega_{\Lambda}=0.7$.

\section{SAMPLE AND METHOD}

The bulk of our sample is drawn from the $\sim 2 \mathrm{deg}^{2}$ COSMOS survey (Scoville et al. 2007). We use photometric redshifts calculated from 30-band photometry by Ilbert et al. (2009); comparison with spectroscopic redshifts shows excellent accuracy. We use those redshifts to derive rest-frame quantities and stellar masses by using the observed broadband photometry in conjunction with a non-evolving template library derived using a Pégase stellar population model (see Fioc \& Rocca-Volmerange 1999) and a Chabrier (2003) stellar initial mass function (IMF). The use of a Kroupa et al. (1993) or a Kroupa (2001) IMF would yield similar stellar masses to within $\sim 10 \%$. The reddest templates are produced through single exponentially declining star formation episodes, the intermediate templates also have a low-level constant star formation rate, and the bluer templates have superimposed a recent burst of star formation. A full description of the stellar masses will be provided in a future paper; comparison with Pannella et al. (2009) masses shows agreement at the 0.1 dex level.

To combat the sample variance of a single $2 \mathrm{deg}^{2}$ field, we augment the COSMOS sample with a sample drawn from three widely separated $0.25 \mathrm{deg}^{2}$ fields from COMBO-17. COMBO-17 photo-z's, colors, and stellar masses have been extensively described in Wolf et al. (2003), Wolf et al. (2004), Borch et al. (2006), and Gray et al. (2009). Given the different depths of the two surveys, we only include galaxies from the COMBO-17 catalog at $z<0.8$, where it is complete for our mass limit. The final sample comprises $\sim 18,000$ galaxies with $M_{*} \geqslant 5 \times 10^{10} M_{\odot}$ over an area of $\sim 2.75 \mathrm{deg}^{2}$ in the redshift range $0.2<z<1.2$.

We use the fraction of galaxies with a companion closer than $30 \mathrm{kpc}$ (close pairs) as a proxy for the merger fraction, as those systems are very likely to merge in a few hundred Myr. ${ }^{6}$ As redshift errors translate into line-of-sight (LOS) distance uncertainties on the order of $\sim 50-100 \mathrm{Mpc}$, we use projected $2 \mathrm{pcf}$ to find the number of projected close pairs and then deproject into the three-dimensional (3D) space.

The projected correlation function $w\left(r_{P}\right)$ is the integral along the LOS of the real-space correlation function:

$$
w\left(r_{p}\right)=\int_{-\infty}^{\infty} \xi\left(\left[r_{p}^{2}+\pi^{2}\right]^{1 / 2}\right) d \pi,
$$

where $r_{p}$ is the distance between the two galaxies projected on the plane of sky and $\pi$ the LOS separation. A convenient

\footnotetext{
6 As the lower mass limit of our sample is $5 \times 10^{10} M_{\odot}$, we are automatically selecting both members of the pair to be above that mass.
}

and simple estimator of the 2 pcf at small scales is $w\left(r_{p}\right)=$ $\Delta(D D / R R-1)$ (e.g., Bell et al. 2006a; Robaina et al. 2009), where $\Delta$ is the path length being integrated over, $D D\left(r_{P}\right)$ is the histogram of separations between real galaxies, and $R R\left(r_{P}\right)$ is the histogram of separations between galaxies in a randomly distributed catalog. As shown in the literature (e.g., Davis \& Peebles 1983; Li et al. 2006), the real-space 2pcf can be reasonably well fit by a power law. Assuming $\xi(r)=\left(r / r_{0}\right)^{-\gamma}$, then $w\left(r_{p}\right)=C r_{0}^{\gamma} r_{0}^{1-\gamma}$, with $C=\sqrt{\pi}\{\Gamma[(\gamma-1) / 2] / \Gamma(\gamma / 2)\}$. We fit the latter expression to our data to find the parameters $\gamma$ and $r_{0}$ and use them in the real-space 2 pcf to find the fraction of galaxies in close pairs.

As we wish to preserve signal-to-noise ratio, we did not integrate along the entire LOS when calculating $w\left(r_{P}\right)$. Instead we allowed galaxies to form a pair only if the redshift difference was smaller than $\sigma_{\text {pair }}=\sqrt{2} \times \sigma_{z}$, with $\sigma_{z}$ being the redshift error of the primary galaxy. As the photo- $z$ errors follow a Gaussian distribution with width $\sigma_{z}$ (Wolf et al. 2003, 2004), a fraction of the pairs would be missing by simply imposing the LOS criteria. Thus, following Bell et al. (2006a), the fraction of galaxy pairs is

$$
f=\int_{-\sigma_{\text {pair }}}^{\sigma_{\text {pair }}} \frac{1}{\sqrt{2 \pi \sigma_{z}}} e^{-z^{2} / 2 \sigma_{z}^{2}} d z .
$$

Then, $w\left(r_{P}\right)$ is multiplied by $1 / f$ in order to account for missing pairs, in our case a correction factor of 1.19.

Given the 3D correlation function $\xi(r)$, the differential probability of finding a galaxy occupying a volume $\delta V$ at a distance $r$ of another galaxy is $\delta P=n[1+\xi(r)] \delta V$, where $n$ is the number density of secondary galaxies. Then, by a simple integration of this expression, we obtain the probability of a galaxy being within a distance $r_{f}$ of any other galaxy (Patton et al. 2000; Bell et al. 2006a; Masjedi et al. 2006):

$$
P\left(r \leqslant r_{f}\right)=\int_{0}^{r_{f}} n[1+\xi(r)] d V .
$$

Because $\xi(r)=\left(r / r_{0}\right)^{-\gamma}$ and $\xi(r) \gg 1$ at the small scales, we obtain

$$
P\left(r \leqslant r_{f}\right)=f_{\text {pair }}=\frac{4 \pi n}{3-\gamma} r_{0}^{\gamma} r_{f}^{3-\gamma},
$$

where $f_{\text {pair }}$ is the fraction of galaxies in close pairs. As galaxy interactions are completely decoupled from the Hubble flow, we calculate probabilities as a function of the proper (physical) separation between the two galaxies. Errors in the correlation function are calculated by means of bootstrap resampling.

\section{RESULTS AND DISCUSSION}

This work aims at an understanding of the impact of galaxy mergers on the evolution of massive galaxies and the evolution of the red sequence at its massive end. To that end we apply the above method to our sample of galaxies with $M_{*}>5 \times 10^{10} M_{\odot}$, regardless of their optical colors, to find the fraction of galaxies in close physical pairs, which we will use as a proxy for the fraction of galaxies undergoing a galaxy interaction. Hereafter, all reference to close pairs will refer to galaxy pairs with inferred separations smaller than $30 \mathrm{kpc}$ in 3D space. Figure 1 and Table 1 show the result of our 2 pcf analysis.

The projected correlation functions in Figure 1 are shown over the range $10<r_{p} / \mathrm{kpc}<1000$, in physical (not comoving) 


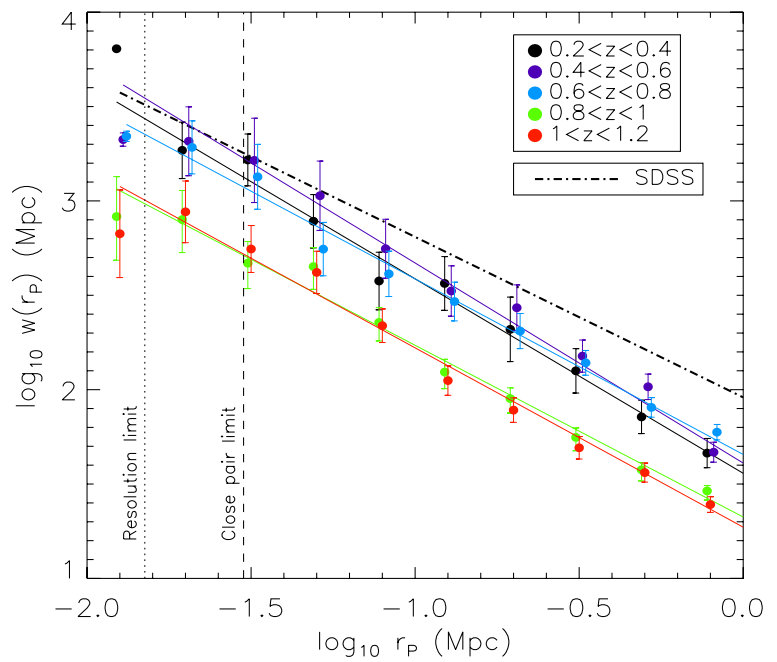

Figure 1. Projected $2 \mathrm{pcf}$ for galaxies with masses $\geqslant 5 \times 10^{10} M_{\odot}$ from our combined COSMOS + COMBO-17 sample in different redshift bins. The bestfit power law is overplotted following the color code of the different redshift bins. The power-law parameters are given in Table 1. The dot-dashed line represents the best-fit power law to the SDSS sample derived from results by $\mathrm{Li}$ et al. (2006). The vertical dashed line represents the separation limit for close pairs of $30 \mathrm{kpc}$ (physical). The vertical dotted line represents the (average of the redshift bins) limit where one can no longer reliably resolve individual galaxies in a pair from the ground-based photometry.

(A color version of this figure is available in the online journal.)

Table 1

3D Correlation Function Parameters and Close Pair Fractions

\begin{tabular}{ccccc}
\hline \hline$z$ & $\gamma$ & $r_{0}$ & $n\left(\mathrm{Mpc}^{-3}\right)$ & $f_{\text {pair }}(<30 \mathrm{kpc})$ \\
\hline $0.2<z \leqslant 0.4^{\mathrm{a}}$ & $2.03 \pm 0.05$ & $3.50 \pm 0.50$ & 0.0031 & $0.0171 \pm 0.0050$ \\
$0.4<z \leqslant 0.6^{\mathrm{a}}$ & $2.06 \pm 0.04$ & $3.60 \pm 0.50$ & 0.0034 & $0.0238 \pm 0.0043$ \\
$0.6<z \leqslant 0.8^{\mathrm{a}}$ & $1.94 \pm 0.04$ & $3.80 \pm 0.30$ & 0.0064 & $0.0241 \pm 0.0038$ \\
$0.8<z \leqslant 1.0^{\mathrm{b}}$ & $1.93 \pm 0.03$ & $2.85 \pm 0.25$ & 0.0131 & $0.0277 \pm 0.0031$ \\
$1.0<z \leqslant 1.2^{\mathrm{b}}$ & $1.96 \pm 0.03$ & $2.55 \pm 0.20$ & 0.0162 & $0.0315 \pm 0.0030$
\end{tabular}

Notes.

${ }^{\text {a }}$ Combined COSMOS+COMBO-17 sample.

${ }^{\mathrm{b}}$ COSMOS—only sample.

units, as is appropriate for a process such as galaxy merging that has decoupled from the Hubble flow. One can see that the fitted power laws are good descriptions of the run of $w\left(r_{p}\right)$ with radius, down to the resolution limit of $\sim 15 \mathrm{kpc}$. Below this radius, we extrapolate the power-law fit. We have checked the accuracy of this extrapolation by comparison with extremely close pairs resolved by Hubble Space Telescope (HST) in the study of two of the four fields used in this paper by Robaina et al. (2009). An extrapolation of our power-law fits to small radius would predict that $\sim 40 \%$ of all our galaxies in $r<30 \mathrm{kpc}$ pairs should have $r<15 \mathrm{kpc}$ separations; we find that $\sim 30 \%$ of galaxies in close pairs have $r<15 \mathrm{kpc}$ separations using $H S T$ data. Accounting for the fact that Robaina et al. (2009) required additional interaction signatures such as tidal tails for a pair to be assigned to that category ${ }^{7}$ and that some extremely close pairs will be classified as already coalesced merger remnants, we

\footnotetext{
7 While most of the projected close pairs at $r<15 \mathrm{kpc}$ are likely to be undergoing an interaction, the morphological signatures do not need to be necessarily evident. Gas fractions and orbital parameters strongly influence the strength of the tidal features. Then, we would expect to find a fraction of extremely close pairs (respect to the total number of close pairs with $r_{p}<30 \mathrm{kpc}$ ) slightly below the expected $40 \%$.
}

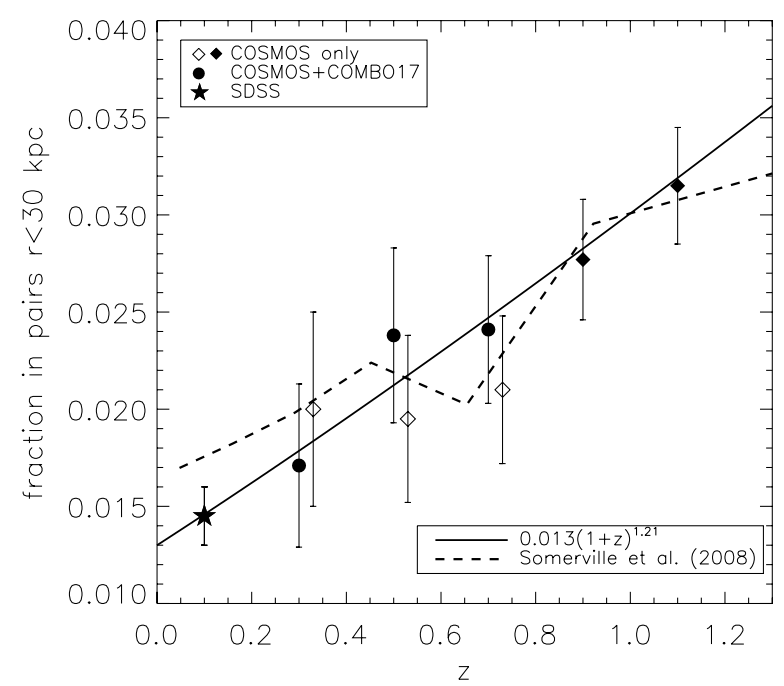

Figure 2. Fraction of $M_{*}>5 \times 10^{10} M_{\odot}$ galaxies in close (3D) pairs $(r<30$ $\mathrm{kpc}$ ) as a function of redshift. Diamonds: pair fraction found using only the COSMOS catalog. Black circles: pair fraction found when adding galaxies from the COMBO-17 survey at $z<0.8$ (we do not include galaxies from the COMBO-17 catalog in the two higher $z$ bins). Star: pair fraction from SDSS. The line shows the best fit to a real-space pair fraction evolution with the shape $F(z)=f(0) \times(1+z)^{m}$, with $f(0)=0.0130 \pm 0.0019$ and $m=1.21 \pm 0.25$ (fit to all black-filled points: star, circles, and diamonds). Dashed line: fraction of galaxies above $M_{*}>5 \times 10^{10} M_{\odot}$ involved in mergers, predicted by Somerville et al. (2008) models when assuming a timescale of $0.5 \mathrm{Gyr}$ to transform from merger rate to fraction.

argue that this level of agreement is remarkable, and we conclude that our extrapolation to radii $r<15 \mathrm{kpc}$ is appropriate. ${ }^{8}$

In Table 1, we show the parameters for the 3D correlation function that we obtain by deprojecting the two-dimensional $2 \mathrm{pcf}$. The evolution of the merger fraction is mainly driven by the evolution in the number density of galaxies above $5 \times 10^{10} M_{\odot}$ in physical coordinates, together with some evolution in the clustering length $r_{0}$.

In Figure 2, we show the fraction of massive galaxies found in pairs with separations $r<30 \mathrm{kpc}$ as a function of $z$. To augment the data at $z \sim 0.1$, we calculate the SDSS close-pair fraction using $\gamma$ and $r_{0}$ given by Li et al. (2006). We adjust their $r_{0}$ by $\sim 5 \%$ (an empirical adjustment based on the COSMOS+COMBO-17 sample) to account for different lower mass limits and binning. We also use the number density of galaxies fulfilling our mass criteria, adopting the $g$-band-selected stellar-mass function in Bell et al. (2003) after correcting for stellar IMF and $H_{0}$.

We perform an error-weighted least-squares fit of the form $F(z)=f(0) \times(1+z)^{m}$ to the filled points in Figure 2, i.e., the SDSS pair fraction, the combined COSMOS+COMBO-17 pair fraction at $0.2<z<0.8$ and the COSMOS pair fraction at $0.8<z<1.2$. We find $f(0)=0.0130 \pm 0.0019$ and $m=1.21 \pm 0.25$. These associated uncertainties are at the same level as the systematic uncertainties in close-pair fraction evolution caused by uncertainty in the overall stellar mass-tolight ratio (M/L) scale and its evolution since $z=1$.

In our correlation function, we do not impose a specific mass ratio criteria, but given the shape of the mass function above

\footnotetext{
8 Note that we could have seemingly sidestepped this issue by using pairs with $15 \mathrm{kpc}<r<30 \mathrm{kpc}$ for the close-pair measurement, and adjusting the timescale to reflect the amount of time pairs spend separated by $15<r / \mathrm{kpc}$ $<30$. Nonetheless, in this case, one would still want to confirm that systems that reached $15 \mathrm{kpc}$ continue to approach smaller radii, amounting to an exercise equivalent to confirming the power-law extrapolation.
} 
$M_{*}>5 \times 10^{10} M_{\odot}$, we expect most of the mergers to be majors, i.e., with mass ratios between 1:1 and 1:4. We measure the fraction of pairs in projection which fulfill such a mass ratio criterion, finding a value between $70 \%$ and $90 \%$ in the different redshift bins.

Galaxies from the COSMOS survey represent $70 \%$ of our sample in the bins ranging from $z=0.2$ to $z=0.8$; however, the addition of COMBO-17 galaxies helps to decrease the sample variance. From the work by Moster et al. (2010), we estimate that the sample variance is reduced by a factor of $\sim 30 \%$ by including the three $\sim 0.25 \mathrm{deg}^{2}$ independent fields from COMBO-17. This effect is clearly seen in Figure 2. Considering only galaxies from COSMOS, there is an abrupt transition between $z=0.7$ and $z=0.9$, which is smoothed by the inclusion of COMBO-17 galaxies.

In Figure 2, we also show the prediction for the close-pair fraction from the semi-analytic models (SAMs) of Somerville et al. (2008), updated as described in Hopkins et al. (2009). The models shown here adopt the best-fit cosmological parameters given in Komatsu et al. (2009). We extract the merger rate of galaxies where both progenitors have stellar masses above $5 \times 10^{10} M_{\odot}$, normalize by the total number density of galaxies above this mass and multiply by 2 to obtain the fraction of galaxies involved in mergers (the quantity that we estimate observationally), per Gyr. Then we multiply by the timescale over which a merger would be observed as a "close pair," which here we assume to be $\tau=0.5$ Gyr following Patton \& Atfield (2008) and Bell et al. (2006a) in order to convert this merger rate into the equivalent of the observed fraction. With this assumed timescale, the model predictions are in excellent agreement with our observational results. Assuming an uncertainty of $~ 30 \%$ in the timescale (matching the numbers from Lotz et al. 2010 for close galaxy pairs timescales) would correspondingly shift vertically the model line by a similar number. Predictions from other SAMs tend to agree reasonably well with those of the Somerville et al. (2008) models, as shown in Figure 11 of Jogee et al. (2009; see also Guo \& White 2008; Hopkins et al. 2010).

\subsection{Comparison with Previous Works}

Very few studies of close-pair fraction evolution use masslimited samples. Bell et al. (2006a) used COMBO-17 data $\left(0.75 \mathrm{deg}^{2}\right.$, the same catalog we use here to complement our COSMOS catalog) to find a fraction of galaxies in close major pairs $(r<30 \mathrm{kpc})$ of $2.8 \%$ for galaxies more massive than $3 \times 10^{10} M_{\odot}$ at $0.4<z<0.8$, in excellent agreement with our result despite the slightly different mass limit. They also performed an autocorrelation of all galaxies with $M_{*}>$ $2.5 \times 10^{10} M_{\odot}$ finding a pair fraction of $\sim 5 \%$. Adopting in our analysis instead a mass limit of $M_{*}>2.5 \times 10^{10} M_{\odot}$, we recover a pair fraction of $\sim 5 \%$. The main driver of this higher pair fraction is the fact that $\sim 50 \%$ of close pairs in a sample limited to have $M_{*}>2.5 \times 10^{10} M_{\odot}$ have mass ratios between $1: 4$ and 1:10, i.e., the major merger fraction is similar, but the close-pair fraction is boosted by a considerable contribution from minors.

Recently, Bundy et al. (2009) performed a similar measurement by studying a sample of galaxy pairs from the GOODS fields (total area $\sim 0.1 \mathrm{deg}^{2}$ ). In Figure 3, we show their results for the mass range $>3 \times 10^{10} M_{\odot}$ after converting their fraction of pairs to the fraction of galaxies in pairs. Bundy et al. (2009) study the fraction of major mergers in galaxies with $M_{*}>3 \times 10^{10} M_{\odot}$, including galaxies $1.5 \mathrm{mag}$ fainter than that mass limit, while by our definition of a close pair, both

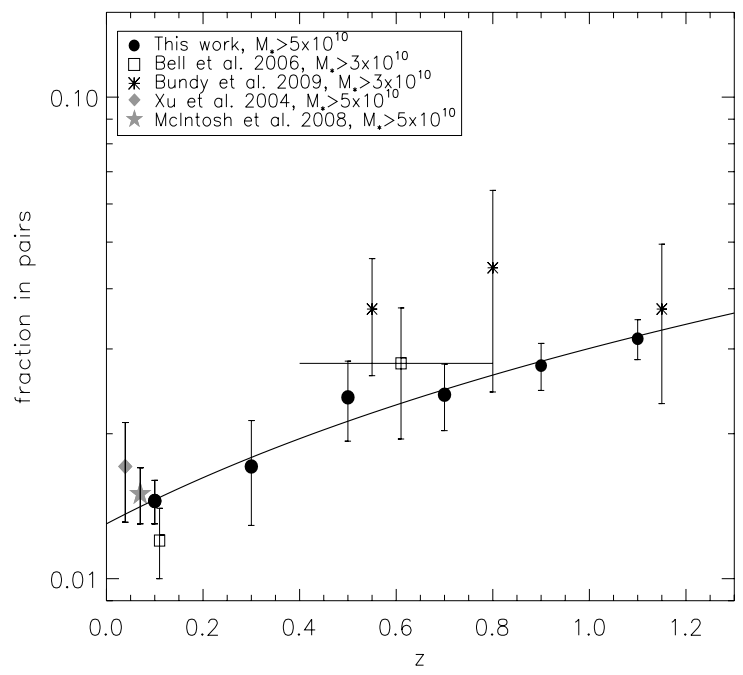

Figure 3. Evolution of the number of galaxies in close pairs. The estimate for the major pair fraction of galaxies $M>3 \times 10^{10} M_{\odot}$ from Bell et al. (2006a) is shown as empty squares. The point by $\mathrm{Xu}$ et al. (2004) is shown as the gray diamond. The point by McIntosh et al. (2008) is shown as the gray star. The results of Bundy et al. (2009) have been corrected down by $33 \%$ in order to match our pair definition (see the text for details).

galaxies need to be above our mass limit. It is natural that they find a higher pair fraction, but we quantify this difference by performing a test in which we try to reproduce the Bundy et al. (2009) methodology. Given the nature of our method, it is difficult to perform a cross-correlation in order to match their pair definition; instead we study the two-dimensional distribution of galaxies in a similar fashion as Bundy et al. (2009). That is, we look for galaxies with separations $r<30 \mathrm{kpc}$ and matched in redshift space and subtract random counts. We perform such a test in the redshift range $0.2<z<0.6$, where we are complete for stellar masses of $\sim 1-2 \times 10^{10} M_{\odot}$, with both our pair definition (both galaxies above $5 \times 10^{10} M_{\odot}$ ) and also keeping such a mass limit for the primary but allowing the secondary to be a factor of 4 less massive. In the first case, we find, as expected, a result compatible with the one found with the $2 \mathrm{pcf}$, and in the second we find that the pair fraction is larger by a factor of $\sim 1.5$. Extrapolating this ratio to the higher redshift bins, we accordingly re-scale the (Bundy et al. 2009) numbers by multiplying their results by 0.67 in order to match our pair definition. The difference is reduced to a factor of less than 2 in the lower redshift bins, while our results basically agree at $z \sim 1$. We also show the estimate from Xu et al. (2004), who used a combined sample from the 2MASS and 2dFGRS surveys, after converting their results to our IMF and $H_{0}$, and the result from McIntosh et al. (2008), who measured the merger fraction in galaxy groups based in projected pairs with additional signs of gravitational interaction. Before performing the comparison, we further correct the pair fractions found by Xu et al. (2004) and Bundy et al. (2009) down by $30 \%$ to account for pairs in their analyses that are genuinely associated with each other (so are not random projections), and have projected separations of $<30 \mathrm{kpc}$ but are separated by more than $30 \mathrm{kpc}$ in real space (i.e., galaxies in groups that are projected along the LOS; Bell et al. 2006a). ${ }^{9}$

We cannot compare our measurements with morphological studies of merger fractions because of uncertainties with the nature of the progenitors (differentiation between major and minor

\footnotetext{
9 This effect is also present in pair fractions determined from spectroscopic redshifts as long as a deprojection to the $3 \mathrm{D}$ space is not performed.
} 
mergers can be extremely challenging, especially in the case in which one of the progenitors is very gas rich), and selection effects related to orbital parameters, galaxy structure, and gas fractions, with the latter introducing a bias in the recovery of interacting systems at higher $z$ (Lotz et al. 2010). A more fundamental difference with respect to morphological studies, both those performed with visual classifications (Jogee et al. 2009) and with automated classifiers (Conselice et al. 2009; Lotz et al. 2008), is the fact that both merger remnants and closely interacting pairs can show signatures of gravitational interactions (see Robaina et al. 2009, for a discussion on the relative numbers of merger remnants and close-pair interactions with morphological disturbances). These two groups are related to different timescales, and the strength and abundance of tidal features are also different (often degenerate with other parameters such as gas fraction). Studies on close-pair statistics aim to detect the galaxy interaction in an early stage, where both progenitors can be studied separately, while morphological studies include systems both before and after the coalescence. For this reason, a direct, quantitative comparison is impossible. It is also hazardous to compare with close-pair measurements based on luminosityselected samples. Lin et al. (2008) used a sample of galaxies with $B$-band magnitudes (corrected for passive luminosity evolution) $-21<M_{B}<-19$. For red galaxies, this is roughly compatible with our mass-selected sample, but it includes many low-mass blue galaxies, which makes a comparison impossible because their clustering properties are very different. As an extreme example, in which one close pair is formed by a very red $M_{B}=-21$ galaxy and a very blue one with $M_{B}=-19$, the stellar-mass ratio in this hypothetical interaction could be as high as 40. This pair definition is clearly not compatible with one in which both galaxies are selected by stellar mass. Furthermore, as merging can enhance the star formation activity (Barton et al. 2000; Robaina et al. 2009), selecting galaxies in rest-frame blue bands is biased in favor of mergers, as such a selection would recover merging systems with lower mass given the decreased $\mathrm{M} / \mathrm{L}$ ratio induced by the interaction. All of these effects will be more pronounced in gas-rich galaxies, and gas fractions were likely higher in the past, leading to a redshift dependent bias.

Kartaltepe et al. (2007) derived the evolution of the pair fraction from a luminosity-selected sample from a similar data set as analyzed in this paper. Their very different result (they find $m=3.1 \pm 0.1$ ) is caused at least in part by the difference between mass- and luminosity-selected samples, as described above, as well as by the fact that they do not correct for passive luminosity evolution. In addition, they identify pairs in both the ground-based and HST/ACS-based catalogs. Because their sample is selected by ground-based luminosity, very close pairs that are only resolved by ACS can be as bright as a single galaxy in a more widely separated pair that is resolved in the groundbased imaging. This artificially raises that close-pair fraction, especially at high redshift.

Summarizing, we choose not to compare with merger fractions obtained by morphological indicators nor with those obtained by close-pair counts in luminosity-selected samples; these provide measurements of different merger-related quantities and cannot, in general, be compared to our measurement.

\subsection{The Impact of Galaxy Merging on the Creation of $M_{*}>10^{11} M_{\odot}$ Galaxies}

Our measured fraction of $M_{*}>5 \times 10^{10} M_{\odot}$ galaxies in close pairs as a function of redshift constrains the impact that merging-induced mass assembly has on the creation rate of $M_{*}>10^{11} M_{\odot}$ systems.

As only $\sim 6 \%$ of our galaxies with a projected companion at $r<30 \mathrm{kpc}$ have a second companion at such separation, we assume that the number of close pairs is simply one half of the number of galaxies in close pairs, neglecting the very minor influence of such a small number of galaxies in triplets. The fraction of newly created $M_{*}>10^{11} M_{\odot}$ galaxies due to merging is $f_{\text {rem }}=N_{\mathrm{cp}} / N_{11}$, where $N_{\mathrm{cp}}$ is the number of close pairs of galaxies above $5 \times 10^{10} M_{\odot}$ each (regardless of their optical colors) and $N_{11}$ is the total number of galaxies with stellar masses in excess of $10^{11} M_{\odot}$.

Following Patton \& Atfield (2008) or Bell et al. (2006a), the merger timescale for galaxy pairs at this separation is approximately $\tau=0.5 \mathrm{Gyr}$, so we compute the creation rate of newly assembled galaxies, $R_{\text {rem }}$, as $R_{\text {rem }}=f_{\text {rem }} / \tau$. We integrate the merger rate over cosmic time finding that, on average, present-day galaxies with stellar masses larger than $10^{11} M_{\odot}$ have undergone $0.5(0.7)$ mergers since $z=0.6$ (1.2) from interactions between galaxies more massive than $5 \times 10^{10} M_{\odot}$.

\subsection{The Merger-driven Evolution of the Red Sequence}

In the previous sections, we have studied the impact of merging on the evolution of the mass function of galaxies, without restriction to the optical colors of the progenitors or descendants. Now, we want to address the question whether our observed merger rate evolution can explain the observed number density evolution in the massive end $\left(M_{*}>10^{11} M_{\odot}\right)$ of the red sequence.

First, we convert the evolution of the $B$-band LF of red galaxies as measured by Brown et al. (2007) to the evolution in the number density of galaxies more massive than $10^{11} M_{\odot}$. For this conversion, we use the typical stellar $\mathrm{M} / \mathrm{L}$ of nearby red galaxies $\left(\mathrm{M} / \mathrm{L}_{B}=3.4 \pm 0.6\right.$, e.g., Bell et al. 2003; Kauffmann et al. 2003) and apply a correction to account for its evolution with redshift, derived from the evolution of the fundamental plane $(\mathrm{FP})$ zero point $\left(\Delta \log \left(\mathrm{M} / \mathrm{L}_{B}\right)=0.555 \pm 0.042\right.$ between $z=1$ and $z=0$; van Dokkum \& van der Marel 2007). We repeat the process 10,000 times allowing the LF parameters, as well as the $\mathrm{M} / \mathrm{L}_{B}$ constraints, to vary randomly within their uncertainties. We estimate the final uncertainty by estimating the typical dispersion of those 10,000 realizations. The results from this exercise are shown in Figure 4. We find an evolution in the number density of the massive end of the red sequence of a factor of $\sim 4$ between $z=1$ and $z=0$.

Our inference of a factor of $\sim 4$ evolution in the number density of red sequence galaxies with $M_{*}>10^{11} M_{\odot}$ is somewhat larger than a number of recent estimates. The main reason for this is our adoption of the recent results by van Dokkum \& van der Marel (2007) for the evolution of M/L determined through FP evolution, who find relatively rapid evolution of $\mathrm{M} / \mathrm{L}$ leading to more rapid inferred fading of the stellar population than assumed by many previous works. For example, Cimatti et al. (2006) found a result compatible with a constant number density of $M_{*}>10^{11} M_{\odot}$ galaxies in the interval $0<z<0.8$. In order to obtain that result, they applied a passive luminosity correction of $\Delta \log \left(\mathrm{M} / \mathrm{L}_{B}\right)=0.46 \pm 0.04$ to a sample drawn from COMBO-17 and DEEP2 (Davis et al. 2003; Faber et al. 2007). This luminosity correction was obtained from the works by van Dokkum \& Stanford (2003) and Treu et al. (2005) and is lower than the one found a few years later by van Dokkum \& van der Marel (2007) with a larger sample. This apparently small difference in $\Delta \log \left(\mathrm{M} / \mathrm{L}_{B}\right)$ has 


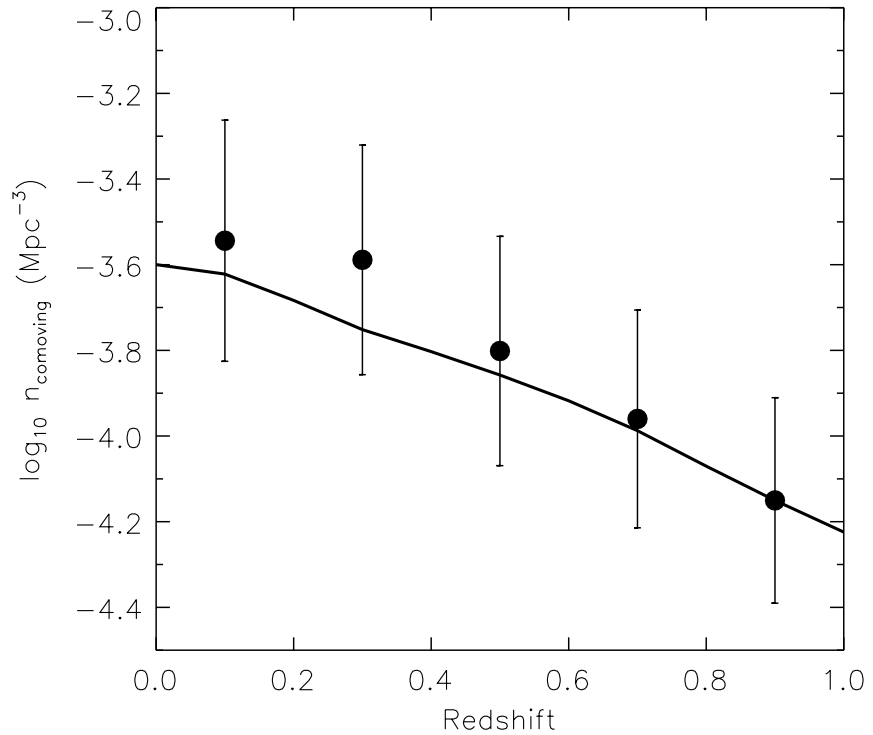

Figure 4. Number density evolution of red galaxies with $M_{*}>10^{11} M_{\odot}$. Filled points with error bars represent the number density observed by Brown et al. (2007) when translated to stellar mass (see the text for details). The solid line shows the expected growth implied by our measurement of the merger rate.

strong implications when studying the number density evolution of massive red galaxies at the level of $\sim 1.5-2$ between $z=1$ and $z=0$. Also, the number of galaxies in their COMBO17 or DEEP2 samples is relatively small when compared to the $\sim 7 \mathrm{deg}^{2}$ of the NOAO Deep Wide-Field Survey (NDWFS; Jannuzi \& Dey 1999) used by Brown et al. (2007). Cool et al. (2008) used a sample of luminous red galaxies (LRGs) from SDSS and spectroscopic follow-up from MMT to address a similar question. They found no evolution in the number density of LRGs since $z=0.9$. We believe that the main driver of the difference between Cool et al.'s result and ours is, again, the correction for $\mathrm{M} / \mathrm{L}$ ratio evolution. They used stellar population models from Bruzual \& Charlot (2003) and Maraston (2005), which give slower evolution than FP zero point evolution constraints.

In a recent work, Matsuoka \& Kawara (2010) studied the number density evolution of massive galaxies since $z \sim 1$ from a sample drawn from UKIRT Infrared Digital Sky Survey and SDSS. Instead of applying an average M/L correction, they calculated stellar masses for individual galaxies at all redshifts, finding a factor of $\sim 4$ evolution at $M_{*}>10^{11} M_{\odot}$ (when adding up their two mass bins). Unlike our present work, they did not differentiate between red and blue galaxies, but given the predominance of red galaxies at such high stellar masses (they report a blue fraction $<30 \%$ at all redshifts in the same mass range) we believe that our factor $\sim 4$ evolution agrees remarkably well with their result.

We now address the question of whether galaxy mergers between massive galaxies can drive the observed number density evolution of massive red galaxies. For that purpose, we use the result for the merger rate found in Section 3.2 and work under the assumption that mergers between massive galaxies will produce remnants with red optical colors. Dry (gas-free) mergers are likely to play an important role in the buildup of the massive end of the red sequence (van Dokkum 2005; Bell et al. 2006b), and the descendant systems of such interactions will be clearly red systems as well. Moreover, mergers involving massive blue, disk-like galaxies are also expected to produce red ellipticals as remnants. Major interactions, independently of the color of the progenitors, are expected to perturb the orbits of the stars in the galaxies and form elliptical systems, but the observed fraction of spheroidal galaxies with blue optical colors is a negligible fraction of the total at masses above $10^{11} M_{\odot}$ (see Schawinski et al. 2009; Kannappan et al. 2009; HuertasCompany et al. 2010). It is likely that even those few systems will passively evolve to the red sequence in $\lesssim 1 \mathrm{Gyr}$, so our assumption that merger remnants with masses above such stellar mass will become red sequence galaxies is justified.

We use the number density of massive systems at $z \sim 0.9$ that we have obtained as the starting point and calculate the growth in the number of massive red galaxies implied by our measurement of the merger rate. We show the result of this exercise as the solid line in Figure 4. We stress that the observed evolution (filled circles) and the one implied by our measurement (solid line) are completely independent except for the fact that we use the observed density at $z \sim 0.9$ to anchor the evolution predicted by our close-pair fractions. We find that mergers of massive galaxies can explain the evolution in the observed number density of massive red galaxies since $z=1$. In other words, the majority of galaxies observed today at the massive end of the red sequence have been assembled in the last $8 \mathrm{Gyr}$ through mergers of massive galaxies.

We have used $\tau=0.5 \mathrm{Gyr}$, but using the $\tau \sim 1$ Gyr timescale from Kitzbichler \& White (2007) produces a somewhat slower evolution that is still compatible within the error bars.

There are two caveats we would like to mention. First, given the nature of our method, some of the progenitor galaxies have masses above $10^{11} M_{\odot}$, so strictly speaking they are not newly formed massive galaxies. Second, because we adopt a lower mass limit of $5 \times 10^{10} M_{\odot}$, we underestimate the number of major mergers that could lead to the formation of a massive galaxy. For example, a pair with individual masses $M_{*}=6 \times 10^{10} M_{\odot}$ and $M_{*}=4 \times 10^{10} M_{\odot}$ would not make it into our pair sample but would produce a major merger remnant of $10^{11} M_{\odot}$. Assuming that the merging population has a composition similar to our overall sample, we estimate that this latter lower mass limit issue has an impact a factor of $\sim 2$ larger on the creation of $>10^{11} M_{\odot}$ galaxies than the overestimate caused by double counting already massive red galaxies (i.e., for every 10 massive galaxies we are counting as produced by mergers, there would be 1-2 which were already above $10^{11} M_{\odot}$ and $\sim 3$ that we are not accounting for because one of the progenitors has a mass below our mass limit of $\left.5 \times 10^{10} M_{\odot}\right)$. This means that our results are uncertain at the level of $\lesssim 20 \%$ when counting newly formed massive red galaxies. We do not apply this correction to our results but would like to stress that, if anything, we slightly underestimate the real creation rate of massive galaxies induced by merging.

Hopkins et al. (2008) found by using Halo Occupation Distribution (HOD) models a merger-driven evolution of a factor of 3-4 in the integrated stellar-mass density in red elliptical galaxies between $z=1$ and $z=0$. Given that we focus on the massive end of the distribution, a quantitative comparison is impossible, but we argue that our results are in qualitative good agreement with the trend seen in that work and in Hopkins et al. (2006), where they found some evolution in $M_{*}>10^{11} M_{\odot}$ red galaxies in the same redshift range by evolving the mass function of red galaxies in a manner compatible with the predicted merger rates.

White et al. (2007) studied the clustering of LRGs in the NDWFS in conjunction with HOD models, finding that $\sim 1 / 3$ of red luminous satellites present at $z=0.9$ have disappeared 
by $z=0.5$. The most likely explanation for this phenomenon is that merging has taken place, decreasing the number of such galaxies, in good qualitative agreement with our result.

Also, in a recent work, van der Wel et al. (2009) studied the shape (axis ratio) distribution of a large sample of quiescent galaxies drawn from the SDSS. Quiescent galaxies with prominent disks are exceedingly rare at stellar masses above $10^{11} M_{\odot}$, providing a strong indication that most, if not all, massive quiescent galaxies form through major mergers. Our results are consistent with this entirely independent result and, moreover, suggest that most of this merger activity occurred over the past $\sim 8 \mathrm{Gyr}$, since $z=1$.

\section{CONCLUSIONS}

We have studied the impact of galaxy mergers on the evolution of massive galaxies by using 2 pcf to measure the fraction of galaxies in close pairs from a sample of $\sim 18,000$ galaxies more massive than $5 \times 10^{10} M_{\odot}$ drawn from the COSMOS and COMBO-17 surveys and a pair fraction estimate from SDSS. We have also used constraints from the observed evolution of the FP zero point to calculate the number density evolution of $M_{*}>10^{11} M_{\odot}$ red galaxies from Brown et al. (2007) LFs. Our main findings are as follows.

1. The fraction of galaxies more massive than $5 \times 10^{10} M_{\odot}$ in close pairs evolves as $F(z)=(0.0130 \pm 0.0019) \times$ $(1+z)^{1.21 \pm 0.25}$ in the redshift range $0<z<1.2$. Assuming a merging timescale of $\tau=0.5 \mathrm{Gyr}$, this implies that galaxies more massive than $10^{11} M_{\odot}$ have undergone, on average, 0.5 (0.7) major mergers since $z=0.6$ (1.2) involving progenitors more massive than $5 \times 10^{10} \mathrm{M}_{\odot}$.

2. The number density of $M_{*}>10^{11} M_{\odot}$ galaxies on the red sequence increases by a factor of $\sim 4$ between $z=1$ and $z=0$. This result strongly depends on the assumed evolution of the $\mathrm{M} / \mathrm{L}$ ratio, which we have assumed here to be $\Delta \log \left(\mathrm{M} / \mathrm{L}_{B}\right)=0.555 \pm 0.042$ between $z=1$ and $z=0$ following recent estimates by van Dokkum \& van der Marel (2007).

3. The evolution implied by our measured merger rate is sufficient to explain this observed number density evolution of massive red galaxies since $z=1$; major mergers between massive $\left(M_{*}>5 \times 10^{10} M_{\odot}\right)$ galaxies are the main driver of the red sequence growth at its massive end.

A.R.R. and E.F.B. acknowledge support through the DFG Emmy Noether Programme. A.R.R. and R.E.S. acknowledge the Heidelberg-International Max Planck Research School program. C.W. was supported by an STFC Advanced Fellowship.

\section{REFERENCES}

Abraham, R. G., et al. 1996, MNRAS, 279, L47

Barton, E. J., Geller, M. J., \& Kenyon, S. J. 2000, ApJ, 530, 660

Bell, E. F., McIntosh, D. H., Katz, N., \& Weinberg, M. D. 2003, ApJS, 149, 289 Bell, E. F., Phleps, S., Somerville, R. S., Wolf, C., Borch, A., \& Meisenheimer, K. 2006a, ApJ, 652, 270

Bell, E. F., Zheng, X. Z., Papovich, C., Borch, A., Wolf, C., \& Meisenheimer, K. 2007, ApJ, 663, 834

Bell, E. F., et al. 2004, ApJ, 608, 752

Bell, E. F., et al. 2005, ApJ, 625, 23

Bell, E. F., et al. 2006b, ApJ, 640, 241
Blanton, M. R., et al. 2003, ApJ, 592, 819

Borch, A., et al. 2006, A\&A, 453, 869

Brown, M. J. I., et al. 2007, ApJ, 654, 858

Bruzual, G., \& Charlot, S. 2003, MNRAS, 344, 1000

Bundy, K., et al. 2009, ApJ, 697, 1369

Chabrier, G. 2003, ApJ, 586, L133

Cimatti, A., et al. 2006, A\&A, 453, L29

Conselice, C. J., Yang, C., \& Bluck, A. F. L. 2009, MNRAS, 394, 1956

Conselice, C., et al. 2003, AJ, 126, 1183

Cool, R. J., et al. 2008, ApJ, 682, 919

Davis, M., \& Peebles, P. J. E. 1983, ApJ, 267, 465

Davis, M., et al. 2003, Proc. SPIE, 4834, 161

De Lucia, G., \& Blaizot, J. 2007, MNRAS, 375, 2

Faber, S. M., et al. 2007, ApJ, 665, 265

Fioc, M., \& Rocca-Volmerange, B. 1999, arXiv:astro-ph/9912179

Gray, M. E., et al. 2009, MNRAS, 393, 1275

Guo, Q., \& White, S. D. M. 2008, MNRAS, 384, 2

Heiderman, A., et al. 2009, ApJ, 705, 1433

Hopkins, P. F., Cox, T. J., Kereš, D., \& Hernquist, L. 2008, ApJS, 175, 390

Hopkins, P. F., Hernquist, L., Cox, T. J., Robertson, B., \& Springel, V. 2006, ApJS, 163, 50

Hopkins, P. F., et al. 2009, MNRAS, 397, 802

Hopkins, P. F., et al. 2010, arXiv:1004.2708

Huertas-Company, M., Aguerri, J. A. L., Tresse, L., Bolzonella, M., Koekemoer, A. M., \& Maier, C. 2010, A\&A, 515, 3

Ilbert, O., et al. 2009, ApJ, 690, 1236

Jannuzi, B. T., \& Dey, A. 1999, in ASP Conf. Ser. 191, Photometric Redshifts and the Detection of High Redshift Galaxies, ed. R. Weymann et al. (San Francisco, CA: ASP), 111

Jogee, S., et al. 2009, ApJ, 697, 1971

Kannappan, S. J., Guie, J. M., \& Baker, A. J. 2009, AJ, 138, 579

Kartaltepe, J. S., et al. 2007, ApJS, 172, 320

Kauffmann, G., et al. 2003, MNRAS, 341, 33

Kitzbichler, M. G., \& White, S. D. M. 2007, MNRAS, 376, 2

Komatsu, E., et al. 2009, ApJS, 180, 330

Kormendy, J., Fisher, D. B., Cornell, M. E., \& Bender, R. 2009, ApJS, 182, 216

Kroupa, P. 2001, MNRAS, 322, 231

Kroupa, P., Tout, C. A., \& Gilmore, G. 1993, MNRAS, 262, 545

Le Fèvre, O., et al. 2000, MNRAS, 311, 565

Li, C., et al. 2006, MNRAS, 368, 21

Lin, L., et al. 2004, ApJ, 617, L9

Lin, L., et al. 2008, ApJ, 681, 232

Lotz, J. M., Jonsson, P., Cox, T. J., \& Primack, J. R. 2010, MNRAS, 404, 590

Lotz, J., et al. 2004, AJ, 128, 163

Lotz, J. M., et al. 2008, ApJ, 672, 177

Maraston, C. 2005, MNRAS, 362, 799

Masjedi, M., et al. 2006, ApJ, 644, 54

Matsuoka, Y., \& Kawara, K. 2010, MNRAS, 405, 100

McIntosh, D. H., et al. 2008, MNRAS, 388, 1537

Moster, B. P., Somerville, R. S., Newman, J. A., \& Rix, H.-W. 2010, arXiv: 1001.1737

Pannella, M., et al. 2009, ApJ, 701, 787

Patton, D. R., \& Atfield, J. E. 2008, ApJ, 685, 235

Patton, D. R., et al. 2000, ApJ, 536, 153

Robaina, A. R., et al. 2009, ApJ, 704, 324

Schawinski, K., et al. 2009, MNRAS, 396, 818

Schweizer, F., \& Seitzer, P. 1992, AJ, 104, 1039

Scoville, N., et al. 2007, ApJS, 172, 38

Somerville, R. S., et al. 2008, MNRAS, 391, 481

Taylor, E. N., et al. 2009, ApJ, 694, 1171

Toomre, A., \& Toomre, J. 1972, ApJ, 178, 623

Treu, T., et al. 2005, ApJ, 633, 174

van der Wel, A., Rix, H.-W., Holden, B. P., Bell, E. F., \& Robaina, A. R. 2009, ApJ, 706, L120

van Dokkum, P. G. 2005, AJ, 130, 2647

van Dokkum, P. G., \& Stanford, S. A. 2003, ApJ, 585, 78

van Dokkum, P. G., \& van der Marel, R. P. 2007, ApJ, 655, 30

Walcher, C. J., et al. 2008, A\&A, 491, 713

White, M., Zheng, Z., Brown, M. J. I., Dey, A., \& Jannuzi, B. T. 2007, ApJ, 655, L69

Wolf, C., et al. 2003, A\&A, 401, 73

Wolf, C., et al. 2004, A\&A, 421, 913

Xu, C. K., Sun, Y. C., \& He, X. T. 2004, ApJ, 603, L73 